\title{
GENERALIZED EXPONENTS VIA HALL-LITTLEWOOD SYMMETRIC FUNCTIONS
}

\author{
R. K. GUPTA
}

The generalized exponents of finite-dimensional irreducible representations of a compact Lie group are important invariants first constructed and studied by Kostant in the early 1960 s. Their actual computation has remained quite enigmatic. What was known ([K] and [Hs, Theorem 1]) suggested to us that their computation lies at the heart of a rich combinatorially flavored theory.

This note announces several results all tied together by Theorem 2.3 below, which selects the natural generalizations of the Hall-Littlewood symmetric functions, rather than the irreducible characters, as the best basis of the character ring. Full details will appear elsewhere.

1. Statement of problem. Let $\mathfrak{g}$ be a complex semisimple Lie algebra with adjoint group $G$. Via the adjoint action, the symmetric algebra $S(\mathfrak{g})$ becomes a graded representation of $G$. Kostant studied this representation in his fundamental paper $[\mathbf{K}]$; his results are well known. $S(\mathfrak{g})=I \otimes H$ is a free module over the $G$-invariants $I$ generated by the harmonics $H$. Moreover, $I$ is a polynomial ring on homogeneous generators of known degrees, and $H=\bigoplus_{p \geq 0} H^{p}$ is a graded, locally finite $\mathfrak{g}$-representation.

Hence, to study the isotypic decomposition of $S(\mathfrak{g})$, one forms for each irreducible $G$-representation $V$ the polynomial in an indeterminate $q$ :

$$
F(V):=\sum_{p \geq 0}\left\langle V, H^{p}\right\rangle q^{p}
$$

Here $\langle$,$\rangle is the usual form \operatorname{dim} \operatorname{Hom}_{\mathfrak{g}}($,$) on the representation ring of \mathfrak{g}$. Kostant's problem asks us to determine $F(V)$; he called the integers $e_{1}, \ldots, e_{s}$ with $F(V)=\sum_{i=1}^{s} q^{e_{i}}$ the generalized exponents of $V$.

The polynomial $F(V)$ turns out to be a rather deep invariant of the representation $V$. For instance, the $F(V)$ are certain Kazhdan-Lusztig polynomials for the affine Weyl group (combine [Hs, Theorem 1] and [Ka, Theorem 1.8]), and they describe a certain group cohomology [FP, Theorem 6.1]).

2. A bilinear form. Our idea is to interpret $F$ as a bilinear form on the character ring $\Lambda$ of $\mathfrak{g}$. Precisely, define a $\mathbf{Z}[q]$-valued symmetric bilinear form $\langle\langle\rangle$,$\rangle on \Lambda[q]$ by setting

$$
\left\langle\left\langle\operatorname{ch}\left(V_{1}\right), \operatorname{ch}\left(V_{2}\right)\right\rangle\right\rangle:=F\left(V_{1} \otimes V_{2}^{*}\right),
$$

Received by the editors July 28, 1986 and, in revised form, December 10, 1986.

1980 Mathematics Subject Classification (1985 Revision). Primary 22E46, 17B10, 05A15; Secondary 05A17.

Research supported by a NATO Postdoctoral Fellowship.

(C) 1987 American Mathematical Society $0273-0979 / 87 \$ 1.00+\$ .25$ per page 
for any two g-representations $V_{1}$ and $V_{2}$, and extending $q$-bilinearly. (Here $\operatorname{ch}(V)$ and $V^{*}$ mean the character and dual of $V$.) Our (2.1) makes sense as (1.1) actually defines $F$ on any representation of $\mathfrak{g}$.

We will present a basis in which our new form $\langle\langle\rangle$,$\rangle diagonalizes. First$ fix a Cartan subalgebra $\mathfrak{h}$ of $\mathfrak{g}$ and some familiar associated objects. Let $\Phi$ be the root system with $\Phi^{+}$a choice of positive roots. Form the lattice $P$ of integral weights and its subset $P++$ of dominant ones. Let $W$ be the Weyl group with length function $l$. Set

$$
t_{\pi}(q):=\sum_{\substack{w \in W \\ w \cdot \pi=\pi}} q^{l(w)}, \quad \text { for } \pi \in \mathcal{P} .
$$

Use exponential notation for characters.

Define, for $\lambda \in P^{++}$, the Hall-Littlewood characters

$$
P_{\lambda}:=t_{\lambda}(q)^{-1} \sum_{w \in W} w\left(e^{\lambda} \prod_{\varphi>0} \frac{1-q e^{-\varphi}}{1-e^{-\varphi}}\right) .
$$

These characters are the classical Hall-Littlewood symmetric functions (see $[\mathbf{M}, \mathrm{III}])$ when $\mathfrak{g}=\mathfrak{s l}_{n}$; they appear in this more general context in work of Kato $[\mathbf{K a}]$.

THEOREM 2.3. The $P_{\lambda}, \lambda \in \mathcal{P}^{++}$, form an orthogonal $\mathbf{Z}[q]$-basis of $\Lambda[q]$ with respect to the form $\langle\langle\rangle$,$\rangle , and$

$$
\left\langle\left\langle P_{\lambda}, P_{\lambda}\right\rangle\right\rangle=t_{0}(q) / t_{\lambda}(q) .
$$

We prove this by comparing $\langle\langle\rangle$,$\rangle to \langle$,$\rangle via the expansion \sum_{p \geq 0} \operatorname{ch}\left(H^{p}\right) q^{p}$ $=t_{0}(q) \prod_{\varphi}\left(1-q e^{\varphi}\right)^{-1}$, as we know $[\mathbf{G 1}$, Theorem 2.5] the basis of $\Lambda[[q]]$ dual to $\left\{P_{\lambda}\right\}_{\lambda \in \mathcal{P}++}$ with respect to $\langle$,$\rangle .$

Kato [Ka] expressed the irreducible characters $\chi_{\pi}=\operatorname{ch}\left(V_{\pi}\right), V_{\pi}$ the $\mathfrak{g}$-representation of highest weight $\pi \in P^{++}$, in terms of the $P_{\lambda}: \chi_{\pi}=$ $\sum_{\lambda \in \mathcal{P}++} m_{\pi}^{\lambda}(q) P_{\lambda}$. The polynomials $m_{\pi}^{\lambda}(q)$ are Lusztig's $q$-analogs of $\lambda$ weight multiplicity in $V_{\pi}[\mathbf{L}]$; they satisfy $m_{\pi}^{\lambda}(1)=\operatorname{dim}\left(V_{\pi}^{\lambda}\right)$. We get

Corollary 2.4. For $\alpha, \beta \in \rho^{++}$,

$$
F\left(V_{\alpha} \otimes V_{\beta}^{*}\right)=\left\langle\left\langle\chi_{\alpha}, \chi_{\beta}\right\rangle\right\rangle=\sum_{\theta \in \mathcal{P}++} m_{\alpha}^{\theta}(q) m_{\beta}^{\theta}(q) t_{0}(q) / t_{\theta}(q) .
$$

As Kostant $[\mathbf{K}]$ proved $\left.F(V)\right|_{q=1}=\operatorname{dim}\left(V^{0}\right)$ for all $V$, our formula is a " $q$-analog" of the fact

$$
\left.F\left(V_{\alpha} \otimes V_{\beta}^{*}\right)\right|_{q=1}=\sum_{\theta \in \mathcal{P}++} \operatorname{dim}\left(V_{\alpha}^{\theta}\right) \operatorname{dim}\left(V_{\beta}^{\theta}\right) \#(W \cdot \theta) .
$$

3. Combinatorics of mixed-tensor $\mathrm{SL}_{n}$-representations. We set $\mathfrak{g}=$ $\mathfrak{s l}_{n}$ to illustrate the effective use of $\S 2$ in evaluating $F$ on irreducibles.

We have formulated a stability theory (1981) for the generalized exponents based on a "mixed-tensor" parameterization $V_{\alpha, \beta}^{[n]}$ of the irreducible $\mathrm{PGL}_{n}$ representations, using certain pairs $\alpha, \beta$ of partitions. First we discuss combinatorics of $\mathrm{SL}_{n}$-representations. 
The Weyl group of $\mathrm{SL}_{n}$ is the symmetric group $S_{n} ; \Lambda$ is the ring of symmetric functions in $x_{i}=\exp \left(t_{i}\right), 1 \leq i \leq n$, for $t_{i}$ the coordinates on diagonal matrices in $\mathfrak{s l}_{n}$. $p++$ identifies with the set of partitions of less than $n$ rows via $\sum_{i=1}^{n-1} c_{i} t_{i} \leftrightarrow\left(c_{1}, \ldots, c_{n-1}\right)$. (Note $t_{1}+\cdots+t_{n}=0$.) Then, $\chi_{\lambda}=s_{\lambda}\left(x_{1}, \ldots, x_{n}\right)$, the classical Schur function, and $P_{\lambda}=P_{\lambda}\left(x_{1}, \ldots, x_{n} ; q\right)$. See $[M, I(3), \operatorname{III}(2.6)]$ for the combinatorial theory.

Write partitions $\gamma$ as nonincreasing sequences $\gamma=\left(\gamma_{1}, \gamma_{2}, \ldots\right)$, ignoring trailing zeroes, with length $l(\gamma)=\#\left\{i \mid \gamma_{i} \neq 0\right\}$ and magnitude $|\gamma|=\gamma_{1}+$ $\gamma_{2}+\cdots=\operatorname{degree}\left(s_{\gamma}\right)$. Also write $V_{\gamma}^{[n]}$, rather than $V_{\gamma}$.

Then $m_{\lambda}^{\mu}(q)=0$ unless $|\lambda|-|\mu|=k n$, some $k$, in which case $m_{\lambda}^{\mu}(q)=$ $K_{\lambda, \pi}(q)$, the Kostka-Foulkes polynomial attached to Young tableaux of shape $\lambda$ and weight $\pi=\mu+\left(k^{n}\right)$, by [M, III, 6, Example 3].

Given partitions $\alpha$ and $\beta$ with $l(\alpha)+l(\beta) \leq n$, we defined $V_{\alpha, \beta}^{[n]}$ as the Cartan piece in $V_{\alpha}^{[n]} \otimes\left(V_{\beta}^{[n]}\right)^{*}$, i.e., the irreducible $\mathfrak{s l}_{n}$-component generated by the tensor product of the highest weight vectors in each factor. It follows that $V_{\alpha, \beta}^{[n]}=V_{\gamma}^{[n]}$ for $\gamma$ the componentwise sum (put $s=l(\alpha), t=l(\beta)$ ):

$$
\gamma=\operatorname{prt}_{n}(\alpha, \beta):=(\alpha_{1}, \ldots, \alpha_{s}, \underbrace{0, \ldots, 0}_{n-s-t},-\beta_{t}, \ldots, \beta_{1})+(\underbrace{\beta_{1}, \ldots, \beta_{1}}_{n}) .
$$

For example, $\mathbf{C}=V_{(0),(0)}^{[n]}$, and $\mathfrak{g}=V_{(1),(1)}^{[n]}$.

LEMMA 3.1. Fix $n \geq 1$. Then the $V_{\alpha, \beta}^{[n]}$, where $\alpha$ and $\beta$ satisfy $l(\alpha)+$ $l(\beta) \leq n$ and $|\alpha|=|\beta|$, form an exhaustive, repetition-free list of the irreducible finite-dimensional representations of $\mathrm{PGL}_{n}$.

4. Stability for $\mathrm{PGL}_{n}$ harmonics. Stability was our original reason for foming the $V_{\alpha, \beta}^{[n]}$. Write $H_{n}^{p}$ for the degree $p$ harmonics.

THEOREM 4.1. Fix $p \geq 0$. Then the number of irreducible $\mathrm{PGL}_{n}$-components of $H_{n}^{p}$ is constant for $n \geq 2 p$. Moreover, the decomposition stabilizes: $V_{\alpha, \beta}^{[n]}$ occurs in $H_{n}^{p}$ only when $r=|\alpha|=|\beta| \leq p$, and $\left\langle V_{\alpha, \beta}^{[n]}, H_{n}^{p}\right\rangle$ stabilizes for $n \geq p+r$. Thus, for some finite set $J^{p}$ of partition pairs of common magnitude and some integers $c_{\alpha, \beta}^{p}$,

$$
H_{n}^{p} \simeq \bigoplus_{(\alpha, \beta) \in J^{p}} c_{\alpha, \beta}^{p} V_{\alpha, \beta}^{[n]}, \quad \text { for } n \geq 2 p
$$

Our original proof worked by a combinatorial analysis of the pieces in $S\left(\right.$ End $\left.\mathbf{C}^{n}\right)$ using the Cauchy and Littlewood-Richardson rules. We, R. Stanley, and $\mathrm{P}$. Hanlon then studied the stable series $\lim _{n \rightarrow \infty} F\left(V_{\alpha, \beta}^{[n]}\right)$. See $[\mathbf{S}$, Hn, and G2].

The key question raised by 4.1 is the determination of the $F\left(V_{\alpha, \beta}^{[n]}\right)$ as functions of two variables $q$ and $n$ (with $n \geq l(\alpha)+l(\beta)$ always implicit).

For each value of $n, F\left(V_{\alpha, \beta}^{[n]}\right) \in \mathbf{Z}[q]$ is controlled by the partitions $\lambda=$ $\operatorname{prt}_{n}(\alpha, \beta)$ and $\mu=\left(\beta_{1}^{n}\right)$ of magnitude $n \beta_{1}$. Precisely, $F\left(V_{\alpha, \beta}^{[n]}\right)=K_{\lambda, \mu}(q)$ (this follows by combining [Hs, Theorem 1] with [M, III, 6, Example 3]). 
However, in $\S 5$ we prove that $F\left(V_{\alpha, \beta}^{[n]}\right)$ as a function of $q$ and $n$ is really "controlled" just by $\alpha$ and $\beta$ (symmetrically, as $F\left(V_{\alpha, \beta}^{[n]}\right)=F\left(V_{\beta, \alpha}^{[n]}\right)$ ). Given a partition $\alpha$, let $h_{1}(\alpha), \ldots, h_{r}(\alpha)$ be its hook numbers and $\tilde{\alpha}$ its conjugate partition (see $[\mathbf{M}, \mathrm{I}, 1]$ ). Set $e(\alpha):=\sum_{i \geq 1} i \alpha_{i}$. Previously, we knew only

Proposition 4.2. Assume $|\alpha|=r$.

(i) If $\beta=\left(1^{r}\right)$, then

$$
F\left(V_{\alpha, \beta}^{[n]}\right)=q^{e(\tilde{\alpha})} \prod_{i=1}^{r}\left(1-q^{n-r-\tilde{\alpha}_{i}+i}\right) /\left(1-q^{h_{i}(\alpha)}\right) .
$$

(ii) If $\beta=(r)$, then $F\left(V_{\alpha, \beta}^{[n]}\right)=s_{\alpha}\left(q, \ldots, q^{n-1}\right)$.

5. A formula for $F\left(V_{\alpha, \beta}^{[n]}\right)$. Let us extend the $K_{\lambda, \mu}(q)$ to skew-partitions $\alpha / \pi$ (cf. $[\mathbf{M}, \mathrm{I}, 1.5])$. Although the latter are not partitions, they behave as such. The skew-Schur function is defined by $s_{\alpha / \pi}=\sum_{\gamma}\left\langle s_{\pi} s_{\gamma}, s_{\alpha}\right\rangle s_{\gamma}$. Now define $K_{\alpha / \pi, \theta}(q)$ as the coefficient of $P_{\theta}$ in $s_{\alpha / \pi}$. Set

$$
b_{\theta}(q):=\prod_{i \geq 1}(1-q) \cdots\left(1-q^{m_{i}}\right), \quad \text { for } \theta=\left(i^{m_{i}}\right) ; \quad b_{(0)}:=1 .
$$

THEOREM 5.1. Fix $\alpha$ and $\beta$ with $|\alpha|=|\beta|=r$. Then

$$
F\left(V_{\alpha, \beta}^{[n]}\right)=\sum_{\substack{\pi, \theta \\|\pi|+|\theta|=r}}(-1)^{|\pi|} K_{\alpha / \pi, \theta}(q) K_{\beta / \tilde{\pi}, \theta}(q) \frac{\left(1-q^{n}\right) \cdots\left(1-q^{n-l(\theta)+1}\right)}{b_{\theta}(q)} .
$$

To prove this, we express $V_{\alpha, \beta}^{[n]}$ in terms of the $V_{\gamma}^{[n]} \otimes\left(V_{\delta}^{[n]}\right)^{*}$ using essentially a formula of Littlewood, and then apply 2.4 .

Theorem 5.1 leads to new, unified proofs of several old results, among them 4.1, 4.2, and the stable theorem $[S, 8.1]$ proved by Stanley. But mainly, 5.1 gives the first real means for computing the $F\left(V_{\alpha, \beta}^{[n]}\right)$.

COROLlaRY 5.2. For some polynomial $g^{\alpha, \beta}(q, z)$ over $\mathbf{Z}$,

$$
F\left(V_{\alpha, \beta}^{[n]}\right)=\frac{g^{\alpha, \beta}\left(q, q^{n-r+1}\right)}{(1-q) \cdots\left(1-q^{r}\right)} .
$$

Moreover,

$$
\frac{g^{\alpha, \beta}(q, z)}{(1-q) \cdots\left(1-q^{r}\right)}=\sum_{i=0}^{r} c_{i}(q) \frac{\left(1-q^{r-1} z\right) \cdots\left(1-q^{r-i} z\right)}{(1-q) \cdots\left(1-q^{i}\right)}
$$

for some $c_{i}(q) \in \mathbf{Z}[q]$.

We have some conjectures on the form of the $g^{\alpha, \beta}(q, z)$. The examples below, done by hand, are new; the first is an old conjecture. Define

$$
\left[\begin{array}{lll}
c_{1} & \cdots & c_{r} \\
d_{1} & \cdots & d_{r}
\end{array}\right]_{q}:=\frac{\left(1-q^{c_{1}}\right) \cdots\left(1-q^{c_{r}}\right)}{\left(1-q^{d_{1}}\right) \cdots\left(1-q^{d_{r}}\right)}, \quad \text { for } c_{i}, d_{i} \in \mathbf{Z}^{+} .
$$

We refrain from thinking about these unless they are polynomials in $q$. 
EXAMPLE 5.3. If $\alpha=\beta=(2,1)$, then 5.1 yields

$$
F\left(V_{\alpha, \beta}^{[n]}\right)=q^{3}\left[\begin{array}{ccc}
n+1 & n-1 & n-3 \\
1 & 1 & 3
\end{array}\right]_{q}+q^{5}\left[\begin{array}{ccc}
n-1 & n-2 & n-3 \\
1 & 1 & 3
\end{array}\right]_{q}
$$

EXAMPLE 5.4. Let us find $F\left(V_{\gamma}^{[6]}\right)$ when $\gamma=(6,4,1,1)$. Then $\gamma=$ $\operatorname{prt}_{6}(\alpha, \beta)$, for $\alpha=(4,2)$ and $\beta=(2,2,1,1) .5 .1$ gives

$$
\begin{aligned}
F\left(V_{\alpha, \beta}^{[n]}\right)= & q^{9}\left[\begin{array}{ccccccc}
n+2 & n+1 & n-1 & n-2 & n-4 & n-5 \\
1 & 1 & 2 & 2 & 4 & 5
\end{array}\right]_{q} \\
& +q^{12}\left[\begin{array}{ccccccc}
n+2 & n-1 & n-2 & n-3 & n-4 & n-5 \\
1 & 1 & 2 & 2 & 4 & 5
\end{array}\right]_{q} \\
& +q^{15}\left[\begin{array}{cccccc}
n-1 & n-2 & n-2 & n-3 & n-4 & n-5 \\
1 & 1 & 2 & 2 & 4 & 5
\end{array}\right]_{q} \\
& +\left(q^{9}+q^{10}+q^{11}\right)\left[\begin{array}{cccccc}
n & n-1 & n-2 & n-3 & n-4 & n-5 \\
1 & 1 & 2 & 2 & 2 & 5
\end{array}\right]_{q}
\end{aligned}
$$

So, at $n=6, F\left(V_{\pi}^{[6]}\right)=2 q^{9}+3 q^{10}+7 q^{11}+9 q^{12}+13 q^{13}+13 q^{14}+15 q^{15}+$ $12 q^{16}+11 q^{17}+7 q^{18}+5 q^{19}+2 q^{20}+q^{21}$.

ACKNOWLEDGMENT. I warmly thank University of Paris VI, I.H.E.S., and Max-Planck-Institut for their hospitality.

\section{REFERENCES}

[FP] E. M. Friedlander and B. J. Parshall, On the cohomology of algebraic and related finite groups, Invent. Math. 74 (1983), 85-117.

[G1] R. K. Gupta, Characters and the q-analog of weight multiplicity, I.H.E.S. preprint $M / 86 / 6$ (submitted for publication).

[G2] R. K. Gupta, Stability of polynomial powers of $\mathfrak{g l}_{n}$ (in preparation).

[Hn] P. Hanlon, On the decomposition of the tensor algebra of the classical Lie algebras, Adv. in Math. 56 (1985), 238-282.

[Hs] W. H. Hesselink, Characters of the nullcone, Math. Ann. 252 (1980), 179-182.

[Ka] S. Kato, Spherical functions and a q-analogue of Kostant's weight multiplicity formula, Invent. Math. 66 (1982), 461-468.

[K] B. Kostant, Lie group representations on polynomial rings, Amer. J. Math. 85 (1963), 327-404.

[L] G. Lusztig, Singularities, character formulas, and a q-analog of weight multiplicities, Analyse et Topologie sur les Espaces Singuliers (II-III), Astérisque 101-102 (1983), 208-227.

[M] I. G. Macdonald, Symmetric functions and Hall polynomials, Clarendon Press, Oxford, 1979.

[S] R. Stanley, The stable behavior of some characters of $\mathrm{SL}(n, C)$, Linear and Multilinear Algebra 16 (1984), 3-27.

Department of Mathematics, Brown University, Providence, Rhode ISLAND 02912 